\title{
Effective Removal of Dabigatran by Idarucizumab or Hemodialysis: A Physiologically Based Pharmacokinetic Modeling Analysis
}

\author{
Laura Maria Fuhr ${ }^{1} \cdot$ Nina Hanke ${ }^{1} \cdot$ Bernd Meibohm $^{2} \cdot$ Thorsten Lehr $^{1}$ (D)
}

Published online: 4 February 2020

(c) The Author(s) 2020

\begin{abstract}
Background Application of idarucizumab and hemodialysis are options to reverse the action of the oral anticoagulant dabigatran in emergency situations.

Objectives The objectives of this study were to build and evaluate a mechanistic, whole-body physiologically based pharmacokinetic/pharmacodynamic (PBPK/PD) model of idarucizumab, including its effects on dabigatran plasma concentrations and blood coagulation, in healthy and renally impaired individuals, and to include the effect of hemodialysis on dabigatran exposure.

Methods The idarucizumab model was built with the software packages PK-Sim ${ }^{\circledR}$ and $\mathrm{MoBi} \circledast$ and evaluated using the full range of available clinical data. The default kidney structure in $\mathrm{MoBi}{ }^{\circledR}$ was extended to mechanistically describe the renal reabsorption of idarucizumab and to correctly reproduce the reported fractions excreted into urine. To model the PD effects of idarucizumab on dabigatran plasma concentrations, and consequently also on blood coagulation, idarucizumab-dabigatran binding was implemented and a previously established PBPK model of dabigatran was expanded to a PBPK/PD model. The effect of hemodialysis on dabigatran was implemented by the addition of an extracorporeal dialyzer compartment with a clearance process governed by dialysate and blood flow rates.

Results The established idarucizumab-dabigatran-hemodialysis PBPK/PD model shows a good descriptive and predictive performance. To capture the clinical data of patients with renal impairment, both glomerular filtration and tubular reabsorption were modeled as functions of the individual creatinine clearance.

Conclusions A comprehensive and mechanistic PBPK/PD model to study dabigatran reversal has been established, which includes whole-body PBPK modeling of idarucizumab, the idarucizumab-dabigatran interaction, dabigatran hemodialysis, the pharmacodynamic effect of dabigatran on blood coagulation, and the impact of renal function in these different scenarios. The model was applied to explore different reversal scenarios for dabigatran therapy.
\end{abstract}

\section{Introduction}

The prodrug dabigatran etexilate is approved in Europe and the USA for the prophylaxis of stroke and embolism and for the treatment of deep venous thrombosis [1]. Its active agent

Electronic supplementary material The online version of this article (https://doi.org/10.1007/s40262-019-00857-y) contains supplementary material, which is available to authorized users.

Thorsten Lehr

thorsten.lehr@mx.uni-saarland.de

1 Clinical Pharmacy, Saarland University, Campus C2 2, Saarbruecken 66123, Germany

2 Department of Pharmaceutical Sciences, College of Pharmacy, The University of Tennessee Health Science Center, Memphis, TN, USA dabigatran and the main dabigatran metabolite dabigatranacyl-glucuronide reversibly bind to and inhibit thrombin and thereby delay the blood coagulation. The acyl-glucuronide accounts for $10-24 \%$ of the total dabigatran plasma concentration and shows a comparable pharmacodynamic (PD) activity $[2,3]$. The PD effect of dabigatran is directly correlated to its plasma concentration and can be easily assessed with coagulation assays, such as activated partial thromboplastin time (aPTT), diluted thrombin time (dTT), ecarin clotting time (ECT), or thrombin time (TT) [4]. These coagulation assays determine the velocity of blood coagulation and are used to quantify the anticoagulant activity of dabigatran [5]. Although dabigatran administration does not require routine monitoring, these assays, especially dTT and ECT, are important tools to determine the dabigatran anticoagulant activity and therefore the bleeding risk of patients in emergency situations $[6,7]$. 


\section{Key Points}

The first whole-body physiologically based pharmacokinetic model of idarucizumab has been successfully built and evaluated for the prediction of idarucizumab plasma concentrations and fractions excreted to urine in healthy, elderly, and renally impaired individuals. The impact of renal function on the pharmacokinetics of idarucizumab was implemented using the reported creatinine clearance values of the different study populations to mechanistically model the extent of passive renal filtration and renal reabsorption of idarucizumab.

In a physiologically based pharmacokinetic/pharmacodynamic approach, the validated idarucizumab model was extended to describe the idarucizumab-dabigatran interaction and to predict the impact of idarucizumab on dabigatran plasma concentrations and thus on blood coagulation times in healthy individuals, elderly persons, renally impaired individuals, and dabigatran-treated patients. Furthermore, a dialyzer compartment was added, to reproduce and compare the effect of hemodialysis on dabigatran exposure and blood coagulation.

The thoroughly evaluated idarucizumab-dabigatranhemodialysis physiologically based pharmacokinetic/ pharmacodynamic model can now be applied to investigate and predict the outcome of different dabigatran reversal regimens and to develop individualized treatment options for patients with reduced renal function.

In the case of emergency surgeries or life-threatening bleeding events, patients receiving dabigatran treatment need removal of dabigatran and reversal of its anticoagulant effects. As dabigatran shows low plasma protein binding (35\%) [8] and a moderate volume of distribution (60-70 L) [9], it can be extracted by hemodialysis. Four hours of hemodialysis remove approximately $50-60 \%$ of dabigatran from plasma [9]. For a more immediate, complete, and convenient reversal, the humanized monoclonal antibody fragment idarucizumab was developed and approved as a specific antidote.

Idarucizumab binds dabigatran and dabigatran-acyl-glucuronide with very high specificity and affinity $\left(K_{\mathrm{d}}=2.1\right.$ $\mathrm{pmol} / \mathrm{L}$ ) [10]. The pharmacokinetics of idarucizumab are governed by its molecular weight $(\mathrm{MW}=47.8 \mathrm{kDa})$, which limits passive distribution into tissues. The main clearance processes are endocytosis as well as renal metabolism and excretion [11]. Endocytosis is followed by lysosomal degradation throughout the body. The neonatal Fc receptor can rescue whole antibodies from lysosomal degradation by binding to their $\mathrm{Fc}$ region and returning them to circulation
[11], but idarucizumab as an antibody fragment does not contain an Fc region to interact with the Fc receptor. Furthermore, idarucizumab is eliminated via the kidneys, as it is small enough for glomerular filtration [12], which is followed by active uptake into proximal tubule cells by the multi-ligand receptors megalin and cubilin. After binding to megalin or cubilin on the cellular surface, these receptors mediate the endocytosis of idarucizumab into tubular cells, where it undergoes lysosomal degradation into amino acids, which are returned to the circulation [13]. Consequently, idarucizumab is eliminated rapidly from the body, showing a short initial half-life of $45 \mathrm{~min}$ [10]. Following its intravenous administration, idarucizumab strongly binds to dabigatran and its glucuronide in the blood, causing a shift in the dabigatran plasma-tissue ratio that leads to a redistribution of dabigatran from the extravascular space into the plasma. Therefore, a sufficient amount of idarucizumab has to be applied to intercept this redistributing dabigatran, as otherwise the rebounding dabigatran plasma concentrations cause a second increase of its anticoagulant effects.

As idarucizumab [14] as well as dabigatran [15] are largely eliminated via renal metabolism or excretion, altered renal function as a result of increasing age or renal impairment impacts the pharmacokinetics of both drugs, which can also influence their interaction. Prediction of the outcome of dabigatran reversal gains further complexity from the high inter-individual variability of dabigatran plasma concentrations, caused not only by differences in renal function but also by the impact of P-glycoprotein (Pgp) on the absorption of dabigatran etexilate after oral administration [16]. Physiologically based pharmacokinetic (PBPK) modeling can be a helpful tool to generate further insights into the complex underlying mechanisms that determine the pharmacokinetics of idarucizumab, dabigatran, and their interactions and to guide dose recommendations.

The aims of this analysis were (1) to establish a wholebody PBPK model of idarucizumab, (2) to describe and predict the interaction of idarucizumab with dabigatran in healthy and renally impaired individuals, (3) to establish a hemodialysis model for dabigatran, (4) to predict the effects of idarucizumab and hemodialysis on the pharmacodynamics of dabigatran by establishing a PBPK/PD model of dabigatran, and (5) to simulate different treatment scenarios for dabigatran removal.

\section{Methods}

\subsection{Software}

PBPK modeling was performed with the software packages PK-Sim ${ }^{\circledR}$ and $\mathrm{MoBi} \circledast$, using the PBPK platform for whole-body PBPK modeling of large molecules (version 8.0, 
part of the Open Systems Pharmacology Suite, www.opensystems-pharmacology.org). Parameter optimization using the Monte Carlo algorithm and a sensitivity analysis were performed within $\mathrm{MoBi}{ }^{\circledR}$. Digitization of published plasma concentration-time and effect-time profiles of dabigatran and idarucizumab was accomplished using GetData Graph Digitizer (version 2.26.0.20, () S. Fedorov). Pharmacokinetic parameter analysis and plots were created with MATLAB ${ }^{\circledR}$ R2019a (The MathWorks, Inc., Natick, MA, USA).

\subsection{Idarucizumab Physiologically Based Pharmacokinetic Model}

An extensive literature research was performed to gain information on the physicochemical properties as well as the absorption, distribution, metabolism, and excretion processes of idarucizumab and to obtain parameters describing its interaction with dabigatran. Furthermore, published clinical studies were collected to provide concentration-time profiles and fraction excreted to urine data of idarucizumab and dabigatran as well as effect-time profiles of dabigatran. Digitized observed data from these clinical studies were assigned to the training dataset, used for model development, or to the test dataset, used for model evaluation. Parameters that could not be informed from the literature were optimized by fitting the model to the entire training dataset. Details on all utilized clinical studies and on their assignment to a test or training dataset are provided in Table 1 and Table S1 of the Electronic Supplementary Material (ESM).

For the presented PBPK analysis, experimental data of five published clinical trials were available [14, 17-23], including healthy, elderly, and renally impaired Caucasian individuals, healthy Japanese individuals, and a diverse group of patients requiring dabigatran reversal. Idarucizumab was applied intravenously in doses between $20 \mathrm{mg}$ and $8000 \mathrm{mg}$, either alone or in combination with orally applied, steady-state dabigatran (150 mg or $220 \mathrm{mg}$ of dabigatran etexilate). In total, 38 plasma concentration-time and 24 fraction excreted to urine profiles of idarucizumab were available for the idarucizumab PBPK model. Furthermore, the studies provide 15 plasma concentration-time profiles of dabigatran with corresponding effect-time profiles for aPTT, dTT, ECT, and TT. Three additional reports were found for the development of the dabigatran hemodialysis model, providing four plasma concentration-time profiles of dabigatran during dialysis $[9,24,25]$.

Reported values for age, weight, height, and sex were used to simulate the different clinical studies. Renal function was modeled using reported values for creatinine clearance $(\mathrm{CrCl})$. It was assumed that the reported $\mathrm{CrCl}$ equals the glomerular filtration rate (GFR), and individual values for the parameter "specific glomerular filtration rate" were calculated and implemented, normalizing the reported $\mathrm{CrCl}$ values to the kidney weights of the respective PK-Sim ${ }^{\circledR}$ individuals. In cases where $\mathrm{CrCl}$ was not reported, the model used the specific glomerular filtration rate calculated by PK-Sim ${ }^{\circledR}$ based on the individual subject characteristics. Physiological differences associated with aging and Japanese ethnicity were taken into account as predefined in the PBPK modeling platform.

\subsubsection{Renal Handling of Idarucizumab}

To describe the tubular reabsorption and degradation of idarucizumab, the kidney compartment provided within the modeling platform was extended by addition of a proximal tubule compartment in $\mathrm{MoBi}{ }^{\circledR}$, according to a previously developed PBPK model by Balazki et al. [26]. The new model structure describes glomerular filtration of idarucizumab from the blood into the new tubule compartment and subsequent flow from the tubule into the urine, as further outlined in the ESM.

For the implementation of megalin-mediated endocytosis and degradation of idarucizumab in the tubule, an endosomal compartment was added into the intracellular subcompartment of the kidney. This endosomal compartment was scaled according to the approach used in the PK-Sim ${ }^{\circledR}$ protein model, which is further outlined in the ESM. To simplify the renal uptake described in Sect. 1, megalin was implemented as a transporter, mediating the transport of idarucizumab from the tubule lumen directly into the endosomal compartment, as illustrated in Fig. 1c. The transport was described using Michaelis-Menten kinetics:

$v_{0}=\frac{k_{\mathrm{cat}} \cdot[E] \cdot[I]}{K_{\mathrm{m}}+[I]}$,

where $v_{0}$ is the reaction rate, $k_{\text {cat }}$ is the catalytic rate constant that describes the turnover per time, $[E]$ is the transporter concentration, $[I]$ is the idarucizumab concentration, and $K_{\mathrm{m}}$ is the substrate concentration that is needed to reach half of $k_{\text {cat }}[E]=V_{\max }$. Lysosomal degradation of idarucizumab was introduced into the kidney endosome compartment with the default equation used in PK-Sim ${ }^{\circledR}$, as described in the ESM.

\subsection{Idarucizumab-Dabigatran Interaction}

A recently published PBPK model of dabigatran, its prodrug dabigatran etexilate, and its metabolite dabigatran-acylglucuronide [27] was merged with the final idarucizumab model. As it is described that idarucizumab is able to bind dabigatran as well as its glucuronide in a 1:1 stoichiometric ratio [28], both interactions were implemented accordingly (see the ESM).

The formed idarucizumab-dabigatran and idarucizumabdabigatran glucuronide complexes were described with the 


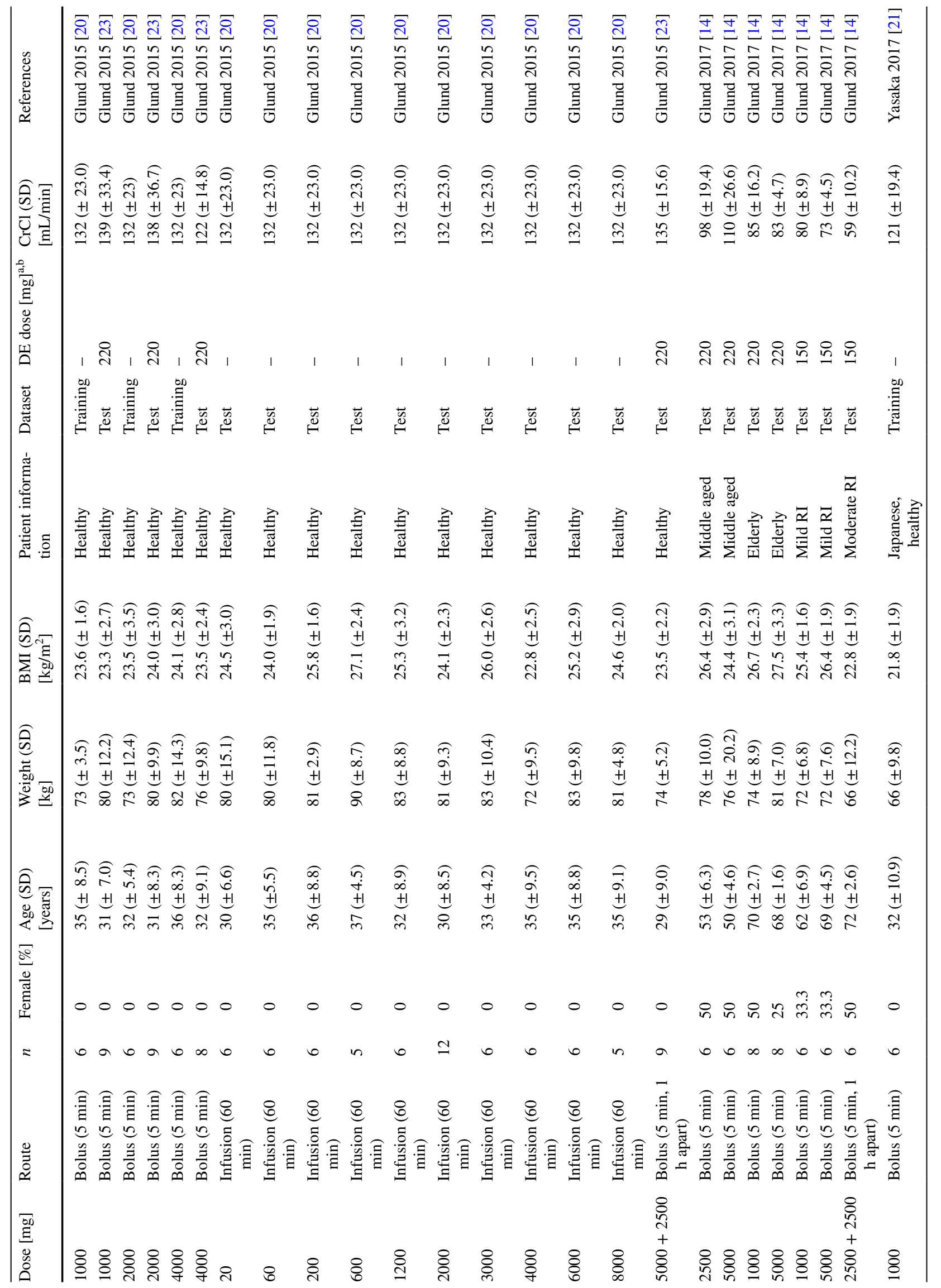




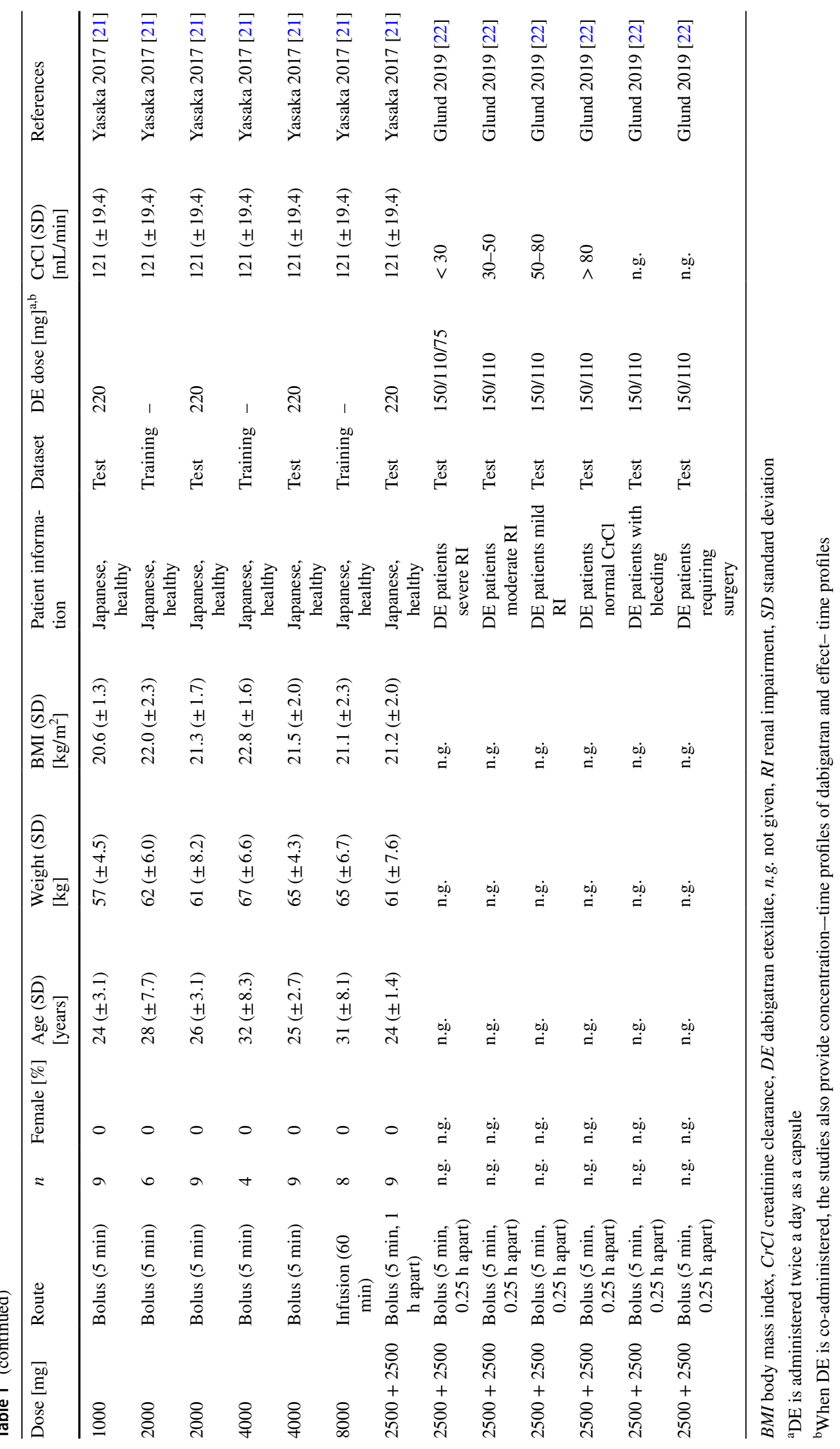


same physicochemical and absorption, distribution, metabolism, and excretion properties as idarucizumab, except for the molecular weights, which have been adjusted. Tubular reabsorption and degradation of the complexes were enabled (see ESM).

In idarucizumab-dabigatran interaction studies, the idarucizumab quantification assay did not differentiate between free idarucizumab and the idarucizumab-dabigatran complexes. Because idarucizumab and the complexes are represented as different molecules in the model, the idarucizumab plasma concentration determined in the clinical studies was described in the model as "sum idarucizumab", by adding up the predicted concentrations of idarucizumab and the idarucizumab-dabigatran complexes.

The dabigatran quantification assay determined the concentration of pharmacologically active dabigatran, which consists of the plasma concentrations of dabigatran and its acyl-glucuronide that are neither bound to plasma proteins nor captured by idarucizumab [14]. Therefore, this measured plasma concentration was described in the model as "unbound sum dabigatran", by adding up the predicted concentrations of unbound dabigatran and unbound dabigatran-glucuronide. A detailed description of dabigatran and idarucizumab plasma and urine measurements and their implementation in the model is provided in Sect. S1.1 of the ESM.

\subsection{Hemodialysis Model}

For hemodialysis modeling, the spatial structure of the human organism within the PBPK modeling platform was extended by a dialyzer compartment in $\mathrm{MoBi}^{\circledR}$, connected to the arterial and venous blood compartments. The filtration of dabigatran and dabigatran glucuronide from venous blood into the dialyzer is described by Eq. (2), adapted from a pharmacometric analysis of dabigatran hemodialysis by Liesenfeld et al. [29]:
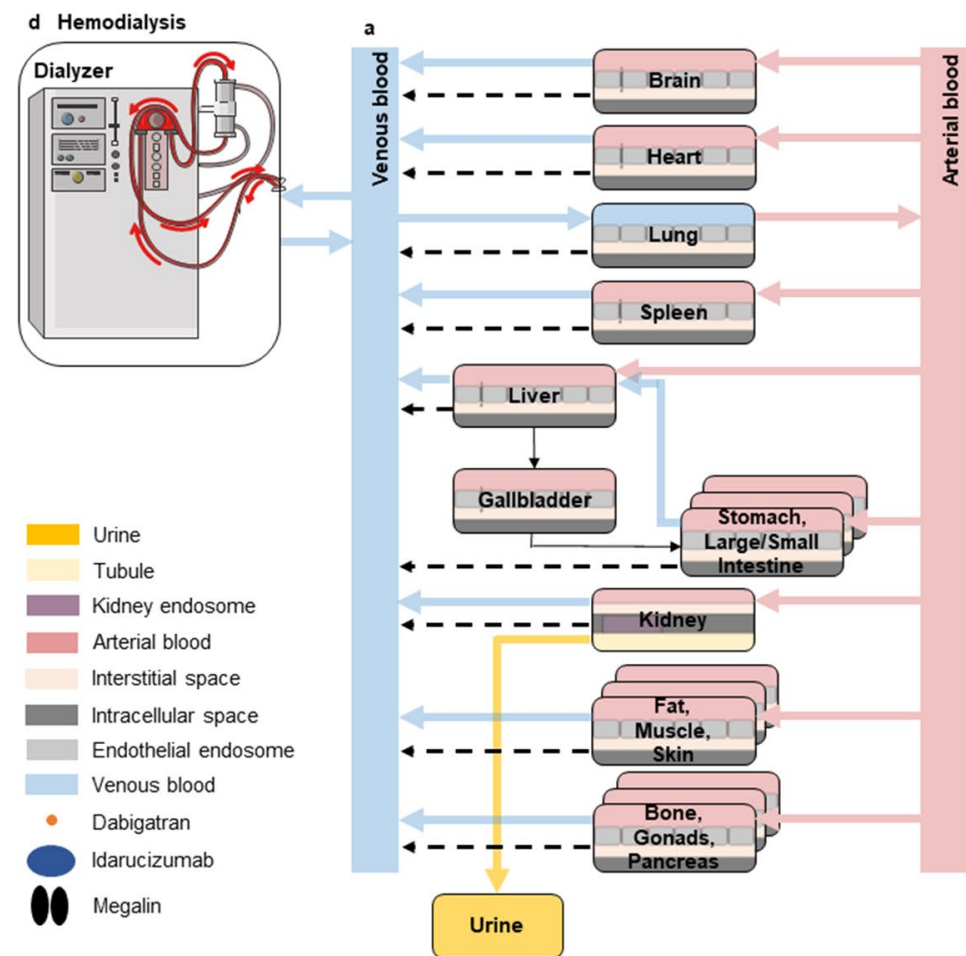
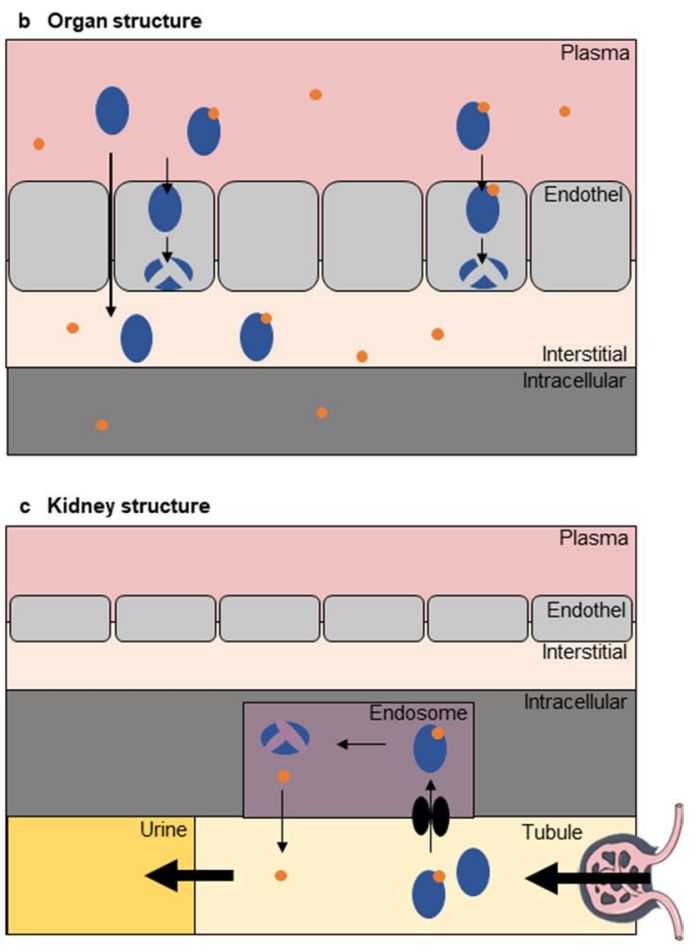

Fig. 1 Final model structure. a The model consists of 17 organ compartments, connected by arterial and venous blood flow (red/blue arrows) and lymph flow (dashed arrows). b Each organ is sub-divided into plasma (also containing red blood cells), endothelial space, interstitial space, and intracellular space. Dabigatran (orange) as a small molecule can distribute freely into all subcompartments, while distribution of the protein idarucizumab (blue) is restricted to plasma and the interstitial space, with the latter accessible via pores in the endothelial cell layer. Therefore, interaction of idarucizumab and dabigatran mainly occurs in plasma and to a lower extent in the interstitial space. Furthermore, idarucizumab enters endothelial cells via endocytosis, where it undergoes lysosomal degradation. c The kidney structure was extended by a tubule compartment and an endosome compartment. Idarucizumab and idarucizumab-dabigatran complexes, filtered from blood into the tubule, are actively transported into the endosome by megalin (black), where they undergo lysosomal degradation. During lysosomal breakdown of the complexes, dabigatran is released and shuttled back into the tubule via exocytosis and excreted with the urine. $\mathbf{d}$ An extracorporeal compartment, representing a dialysis machine, was implemented and connected to the venous blood flow. A clearance process describes the filtration of dabigatran from venous blood into the dialyzer compartment 


$$
\mathrm{CL}_{\text {Dialysis }}=\frac{\mathrm{BFR} \cdot\left(\mathrm{e}^{\frac{\mathrm{KoA}}{\mathrm{BFR}} *\left(1-\frac{\mathrm{BFR}}{\mathrm{DFR}}\right)}-1\right)}{\mathrm{e}^{\frac{\mathrm{KoA}}{\mathrm{BFR}} *\left(1-\frac{\mathrm{BFR}}{\mathrm{DFR}}\right)}-\frac{\mathrm{BFR}}{\mathrm{DFR}}},
$$

where $\mathrm{CL}_{\text {Dialysis }}$ is the dialysis clearance, $\mathrm{BFR}$ is the blood flow rate, DFR is the dialysate flow rate, and KoA is the mass transfer area coefficient for dabigatran, depending on the dialysis filter that is used. KoA was adopted from Liesenfeld et al. [29], while BFR and DFR were taken from the respective publications, shown in Table S1 of the ESM.

\subsection{Dabigatran Pharmacodynamics}

The aPTT, dTT, ECT, and TT assays are performed to determine the PD anticoagulant activity of dabigatran. Because the outcome of these assays depends on the dabigatran plasma concentrations, a concentration-effect relationship of dabigatran and each marker was implemented, to extend the PBPK model to a PBPK/PD model.

Data of the respective coagulation assays were fitted using linear and combined linear and $E_{\max }$ models, to describe their correlation with the dabigatran plasma concentrations, similar to the approach of Liesenfeld et al. [30]. The simplest equation to accurately describe the observed concentration-effect relationship for each marker was then incorporated in the PBPK/PD model.

\subsection{Physiologically Based Pharmacokinetic Model Evaluation}

Predicted plasma concentration-time profiles of idarucizumab and dabigatran and effect-time profiles of dabigatran were compared to the data observed in the clinical studies of the test dataset and further evaluated in goodness-of-fit plots. The performance of the final idarucizumab PBPK model was additionally evaluated by comparison of predicted to observed area under the plasma concentration-time curve (AUC) values. Model prediction was deemed successful when the PK parameters lay within the two-fold acceptance range.

As quantitative measures of the descriptive and predictive performance of the models, mean relative deviation (MRD) of all predicted plasma concentrations and geometric mean fold error (GMFE) of all predicted AUCs were calculated. Calculation of MRD and GMFE is described in the ESM.

\subsection{Prediction of Dabigatran Reversal Scenarios}

After the evaluation of the PBPK models, various reversal scenarios of idarucizumab administration and hemodialysis for dabigatran reversal were predicted. A male Caucasian individual aged 78 years with a body weight of $75 \mathrm{~kg}$ and a
GFR of $62 \mathrm{~mL} / \mathrm{min}$ was created for these predictions, based on preliminary results of the RE-VERSE AD study, reported by Pollack et al. [31]. Administration of $220 \mathrm{mg}$ of dabigatran etexilate twice a day was chosen for all predictions.

\section{Results}

\subsection{Idarucizumab Physiologically Based Pharmacokinetic Model}

The final idarucizumab PBPK model precisely describes and predicts the plasma concentration-time profiles over the entire dosing range reported in the literature, and it furthermore reliably predicts its pharmacokinetics in elderly and renally impaired Caucasian individuals as well as in healthy Japanese individuals. The compartmental structure of the model as well as of the kidney extension are presented in Fig. 1. The final model applies lysosomal degradation of idarucizumab in endothelial cells throughout the body as well as glomerular filtration with subsequent tubular reabsorption via megalin followed by endosomal degradation. Dose-dependent urinary excretion of idarucizumab was observed in vivo and implemented into the model via the Michaelis-Menten kinetics of megalin.

For the description of idarucizumab pharmacokinetics in elderly and renally impaired individuals, changes in kidney function were implemented based on the reported $\mathrm{CrCl}$ values, as described in Sect. 2.2. This resulted in a good description of the idarucizumab plasma concentrations in these individuals, with an under-prediction of the urinary excretion. Adjusting the active uptake of idarucizumab into renal tubule cells of the renally impaired patients, a correlation between the megalin $\mathrm{k}_{\mathrm{cat}}$ and the reported $\mathrm{CrCl}$ could be demonstrated (Fig. S1 and Table S2 of the ESM), and was incorporated into the model using an $E_{\max }$ function (Equation $\mathrm{S} 9$ of the ESM), decreasing megalin $k_{\text {cat }}$ with decreasing $\mathrm{CrCl}$.

When trying to describe the pharmacokinetics of idarucizumab in Japanese individuals, plasma concentrations were under-predicted. By reducing the parameter "GFR fraction" from 0.32 to 0.26 (both values are optimized, as idarucizumab is not freely filtered, owing to its size of $47.8 \mathrm{kDa}$ ) to model the Japanese population, the model performance could be significantly improved, which is reflected by a decrease of the mean MRD by $9 \%$. Predicted vs observed plasma concentration-time profiles using both GFR fractions are presented in Figs. S5 and S6 of the ESM with corresponding MRD values shown in Table S4 of the ESM. The parameters used in the final model are summarized in Table 2.

The good model performance is demonstrated by comparison of predicted to observed plasma concentration-time 
profiles and fraction excreted to urine data in Fig. 2 and Figs. S2-S4 of the ESM. Goodness-of-fit plots and predicted compared to observed AUC values are presented in Fig. 3. Mean predicted plasma concentration MRD and AUC GMFE values (and ranges) are $1.24(1.08-1.56)$ and $1.14(1.00-1.54)$, respectively, with MRD (Table S5 of the ESM) and GMFE (Table S6 of the ESM) values for all individual studies below the prediction acceptance limit of 2, further illustrating the good model performance. A sensitivity analysis showed that the model predictions are sensitive to the value of the GFR fraction and the individual GFR (Fig. S7 of the ESM).

\subsection{Idarucizumab-Dabigatran Interaction}

The model describing the interaction of idarucizumab and dabigatran was established for all populations outlined in Sect. 2.2. The interaction parameters are included in the model parameter table (Table 2).

The final idarucizumab-dabigatran model includes binding of idarucizumab to dabigatran and to dabigatran glucuronide in plasma and the interstitial space. Furthermore, the model applies lysosomal degradation of the resulting complexes in endothelial cells throughout the body, as well as glomerular filtration with subsequent uptake by megalin and degradation in the renal tubules. The idarucizumabbound dabigatran is not degraded in the kidney endosome, but released during the breakdown of idarucizumab and returned to the tubule, where it is excreted with the urine. These processes are illustrated in Fig. 1c and in the ESM.

To account for the highly variable bioavailability of oral dabigatran, simulated dabigatran etexilate doses were adjusted to match the observed data before idarucizumab co-administration. The absolute bioavailability of dabigatran following oral administration of dabigatran etexilate is 3-7\% [1]. Dabigatran etexilate, but not dabigatran, is a substrate of Pgp and the absolute bioavailability of orally administered dabigatran is susceptible to inhibition of Pgp and variability in Pgp expression [32]. The adjusted dabigatran etexilate doses vary between $76 \%(220 \mathrm{mg} \rightarrow 166.11 \mathrm{mg})$ and $107 \%$ (150 mg $\rightarrow 160.13 \mathrm{mg}$ ) of the actually applied doses. This dose adjustment of less than $25 \%$ lies well within the reported inter-individual variability of dabigatran [33].

Plots of predicted compared to observed plasma and urine concentration-time profiles of all idarucizumab-dabigatran interaction studies obtained from the literature are shown in semi-logarithmic plots in Fig. 4 and Figs. S8 and S9 of the ESM, illustrating the good prediction of the drastic reduction of dabigatran plasma concentrations by idarucizumab.

Table 2 Drug-dependent parameters of the final idarucizumab physiologically based pharmacokinetic model

\begin{tabular}{|c|c|c|c|c|c|}
\hline Parameter & Unit & Value used in simulation & Literature value & References & Description \\
\hline \multicolumn{6}{|c|}{ Idarucizumab model } \\
\hline MW & $\mathrm{kDa}$ & 47.8 & 47.8 & {$[20]$} & Molecular weight \\
\hline$f_{\mathrm{u}, \text { plasma }}$ & $\%$ & 100 & 100 & {$[20]$} & Fraction unbound \\
\hline Radius (solute) & $\mathrm{nm}$ & 3.23 & $3.19^{\mathrm{a}}$ & - & Solute radius \\
\hline$K_{\mathrm{d}}(\mathrm{FcRn})$ & $\mu \mathrm{mol} / \mathrm{L}$ & No binding ${ }^{\mathrm{b}}$ & - & - & Dissociation constant \\
\hline$K_{\mathrm{m}}($ megalin $)$ & $\mu \mathrm{mol} / \mathrm{L}$ & 0.20 & - & - & Michaelis-Menten constant \\
\hline$k_{\text {cat }}$ (megalin) & $1 / \min$ & $\begin{array}{l}\left(k_{\text {cat,max }}{ }^{*} \mathrm{GFR}_{\mathrm{IND}}{ }^{n}\right) / \\
\quad\left(\mathrm{GFR}_{50}{ }^{n}+\mathrm{GFR}_{\mathrm{IND}}{ }^{n}\right)^{\mathrm{c}}\end{array}$ & - & - & Catalytic rate constant \\
\hline GFR fraction & - & $0.32 / 0.26^{\mathrm{d}}$ & - & - & $\begin{array}{l}\text { Fraction of GFR used for passive elimina- } \\
\text { tion by the kidney }\end{array}$ \\
\hline $\mathrm{GFR}_{50}$ & $\mathrm{~mL} / \mathrm{min}$ & 73.31 & - & - & GFR needed to reach half of megalin $k_{\mathrm{cat}, \max }$ \\
\hline$k_{\text {cat,max }}$ & $1 / \min$ & 0.05 & - & - & Maximal catalytic rate constant value \\
\hline$n$ & - & 1.64 & - & - & Hill factor \\
\hline \multicolumn{6}{|c|}{ Idarucizumab-DAB interaction model } \\
\hline$K_{\mathrm{d}}(\mathrm{DAB})$ & $\mathrm{pmol} / \mathrm{L}$ & 2.1 & 2.1 & {$[37]$} & Dissociation constant \\
\hline$k_{\text {off }}(\mathrm{DAB})$ & $1 / \mathrm{s}$ & $7 \times 10^{-6}$ & $3 \times 10^{-6}, 7 \times 10^{-6}$ & {$[10,37]$} & Dissociation rate constant \\
\hline$K_{\mathrm{d}}(\mathrm{DABG})$ & $\mathrm{pmol} / \mathrm{L}$ & 2.1 & - & & Dissociation constant \\
\hline$k_{\text {off }}(\mathrm{DABG})$ & $1 / \mathrm{s}$ & $7 \times 10^{-6}$ & - & & Dissociation rate constant \\
\hline
\end{tabular}

$D A B$ dabigatran, $D A B G$ dabigatran glucuronide, $F c R n$ neonatal Fc receptor, $G F R$ glomerular filtration rate, $G F R_{I N D}$ GFR of the simulated individual

${ }^{\mathrm{a} C a l c u l a t e d}$ in PK-Sim ${ }^{\circledR}$

${ }^{\mathrm{b}}$ Idarucizumab has no Fc-region to interact with FcRn

${ }^{\mathrm{c}} k_{\text {cat }}$ calculated individually for each simulation as a function of $\mathrm{GFR}_{\mathrm{IND}}$

${ }^{\mathrm{d}}$ Values for Caucasian and Japanese individuals, respectively 
Fig. 2 Idarucizumab (IDA) plasma concentrations (dark blue) and fractions excreted to urine (fe to urine) (light blue) following administration of different doses $(1000,4000$, or $5000 \mathrm{mg}$ ) as bolus injections to $\mathbf{a}, \mathbf{b}$ healthy Caucasian individuals $[18,20]$; c, d Caucasian individuals between 65 and 80 years of age, pre-treated with $220 \mathrm{mg}$ of dabigatran etexilate (DE) twice a day (bid) [14, 17]; e, f Caucasian individuals with mild renal impairment (RI), pre-treated with $150 \mathrm{mg}$ of DE bid [14, 17], and $\mathbf{g}, \mathbf{h}$ healthy Japanese individuals [19, 21]. Clinically observed data are shown as dots; solid lines indicate predicted plasma concentrations, dashed lines indicate predicted fe to urine
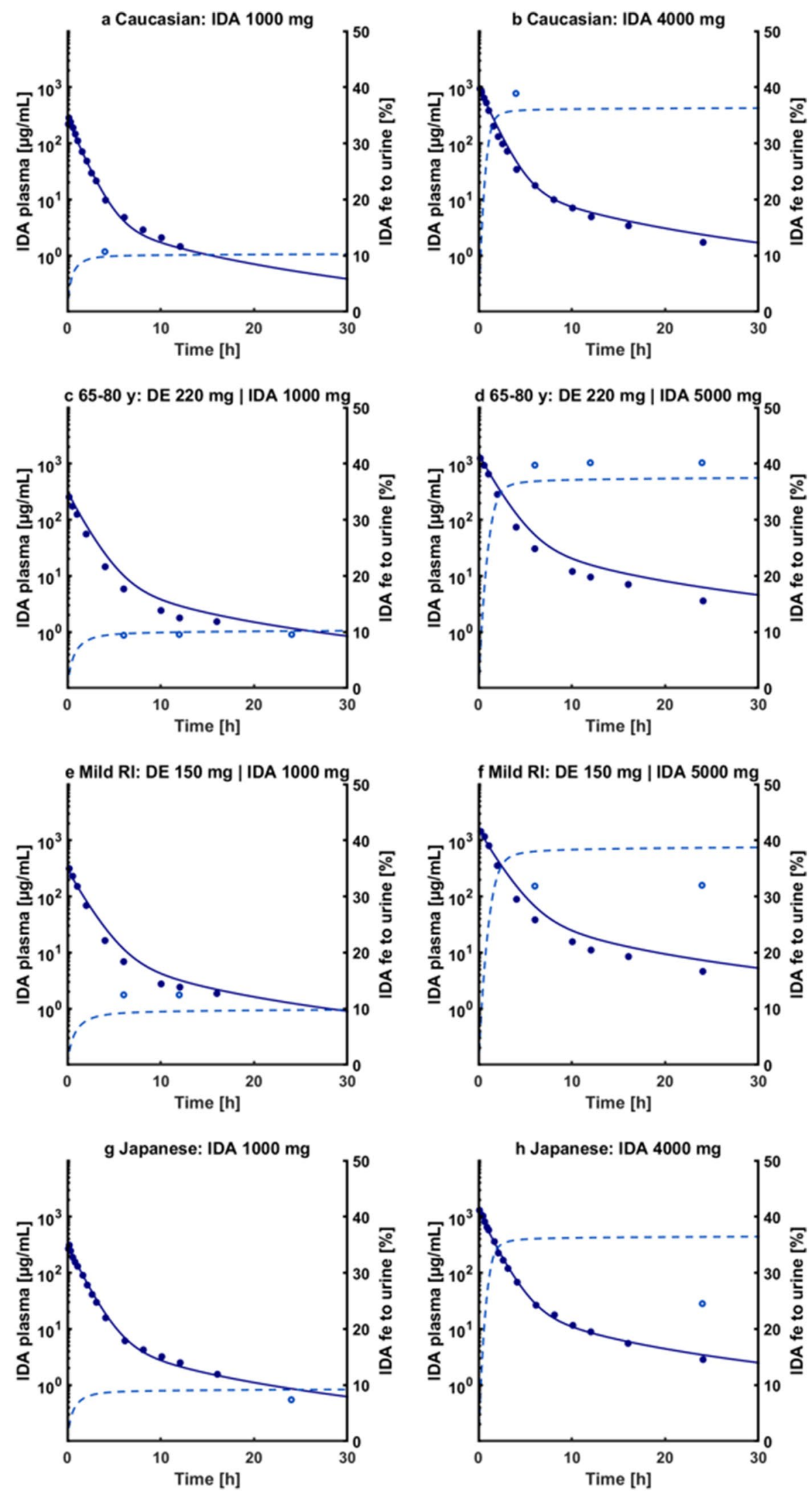

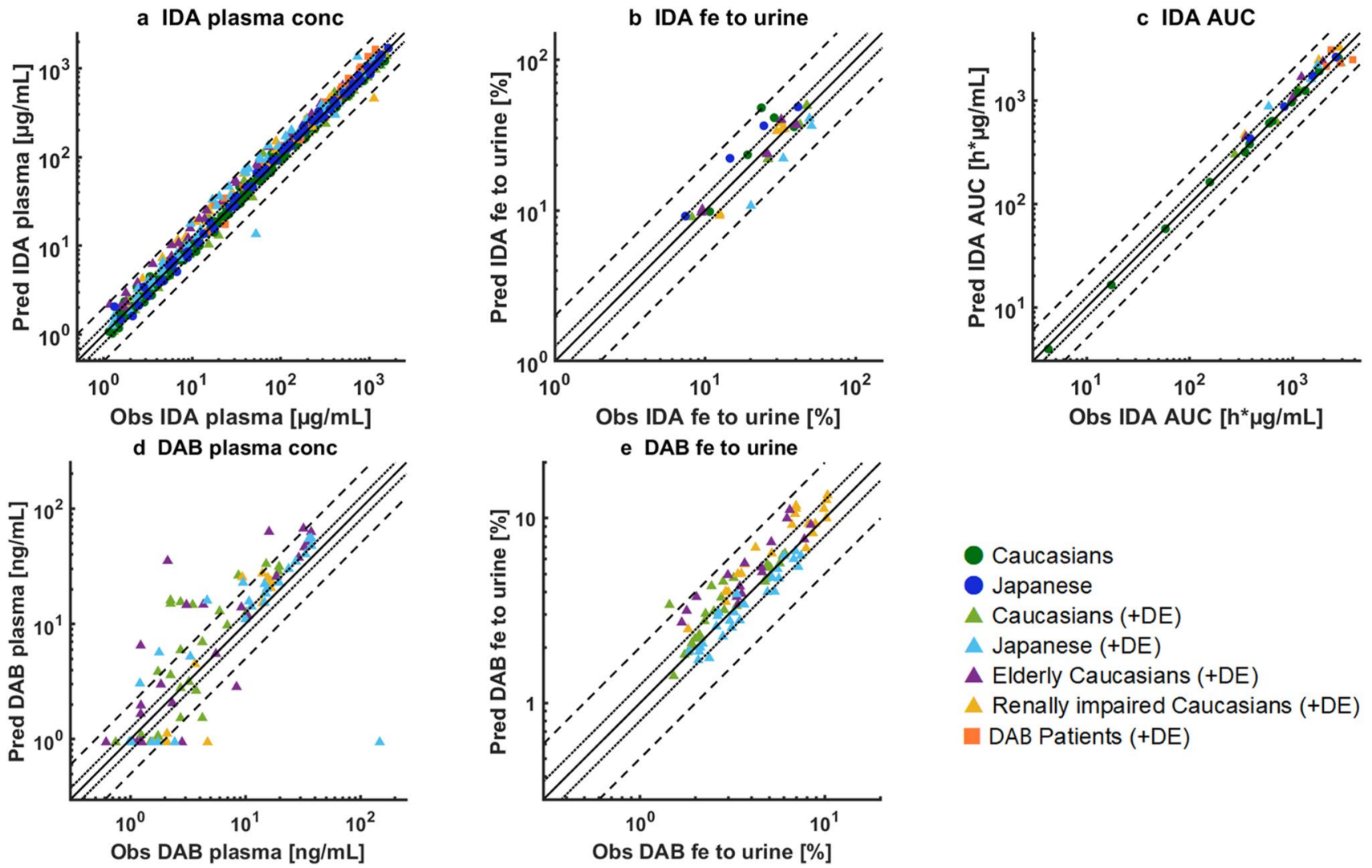

- Caucasians

Japanese

$\triangle$ Caucasians (+DE)

$\triangle$ Japanese $(+D E)$

$\Delta$ Elderly Caucasians (+DE)

$\triangle$ Renally impaired Caucasians (+DE)

DAB Patients (+DE)
Fig. 3 Predicted (pred) compared to observed (obs) a, d plasma concentrations; $\mathbf{b}$, e fractions excreted to urine (fe to urine), and $\mathbf{c}$ areas under the plasma concentration-time curves (AUC), of idarucizumab (IDA) (upper row) and dabigatran (DAB) (lower row). The line

Goodness-of-fit plots of dabigatran plasma concentrations and fractions excreted to urine following idarucizumab administration are presented in Fig. $3 \mathrm{~d}$ and e. $83 \%$ of the predicted plasma concentrations lie within the two-fold range of the observed values with a mean MRD of 2.14 (1.38-5.10).

\subsection{Hemodialysis Model}

To explore the efficiency of hemodialysis for dabigatran reversal, an extension of the dabigatran model was developed by adding a dialyzer compartment and adapting the hemodialysis clearance model established by Liesenfeld et al. [29] for dabigatran and dabigatran glucuronide as described in Sect. 2.4. Concentration-time profiles taken from clinical studies are summarized in Table S1 of the ESM, and predicted compared to observed plasma profiles are shown in Fig. 5. This semi-mechanistic approach successfully predicts the reduction of the sum dabigatran plasma concentrations in different hemodialysis settings, such as different hemodialysis times, blood flow rates, and dialysate flow rates. During hemodialysis sessions of 3,4 , and $6 \mathrm{~h}$, plasma concentration reductions by $41 \%, 49 \%$, and $65 \%$ have been reported, while of identity is shown as a solid line; the 0.8 - to 1.25 -fold bioequivalence limits are shown as dotted lines; the 0.5 - to 2.0 -fold prediction acceptance limits are shown as dashed lines. conc concentration, $D E$ dabigatran etexilate

the presented model predicts reductions by $36 \%, 46 \%$, and $56 \%$, respectively.

\subsection{Dabigatran Pharmacodynamics}

To describe the PD effects of dabigatran, a PBPK/PD model was established, linking the unbound sum dabigatran plasma concentrations to the different coagulation assay results. Following the analysis of experimental data, a combined linear and $E_{\max }$ model was selected to model the correlation of unbound sum dabigatran plasma concentrations to aPTT and TT coagulation times, while a linear model was sufficient to describe the effect of unbound sum dabigatran on dTT and ECT coagulation times. Similar approaches have been described by Liesenfeld et al. [30] and Stangier et al. [3, 6].

The resulting concentration-effect functions are shown in Fig. 6 (left column), with the corresponding equations documented in the ESM. Representative predicted effect-time profiles compared to the respective observed data and goodness-of-fit plots of all predicted compared to observed effect data are also presented in Fig. 6 and demonstrate the good performance of the PBPK/PD model. For aPTT, dTT, ECT, 

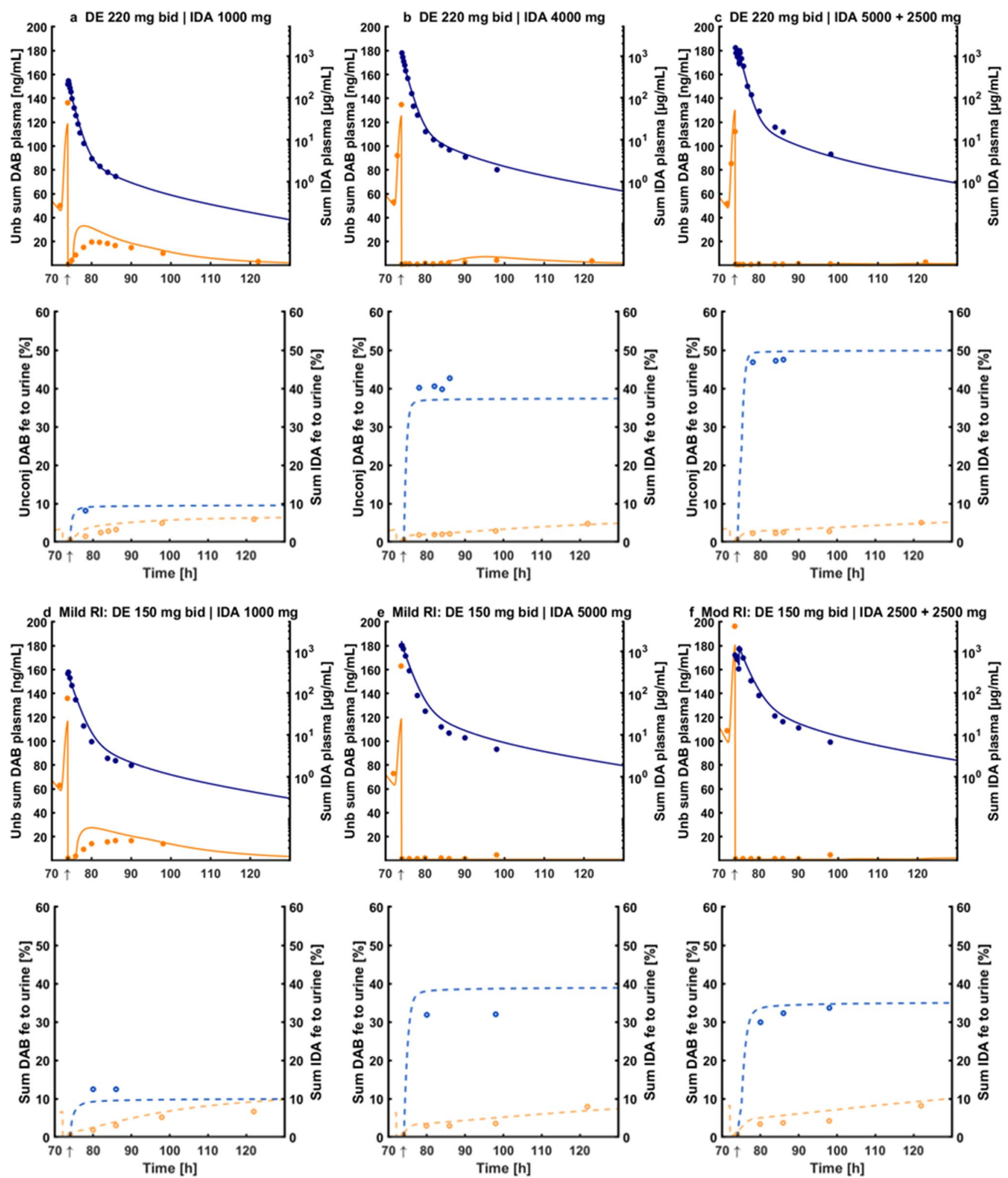

Fig. 4 Plasma concentrations (upper rows) and fractions excreted to urine (fe to urine) (lower rows) of dabigatran (DAB) (orange) and idarucizumab (IDA) (blue) during dabigatran etexilate (DE) treatment (220 mg twice a day (bid), for 3.5 days), followed by administration of different doses of IDA for DAB reversal (1000-7500 mg, at $74 \mathrm{~h}$, arrow) in a-c: healthy Caucasian individuals $[18,23]$ and $\mathbf{d}-\mathbf{f}$ renally impaired Caucasian individuals [14, 17]. Clinically observed data are shown as dots; solid lines and dashed lines indicate the model prediction. mod moderate, RI renal impairment, unb sum unbound sum, unconj unconjugated 
and TT, $98 \%, 99 \%, 98 \%$, and $97 \%$ of the predicted values are within the two-fold range of the observed data, resulting in mean MRD values of $1.16,1.11,1.16$, and 1.27 , respectively. Goodness-of-fit plots as well as MRD calculations include PD measurements before and after the administration of idarucizumab. Mean relative deviation values of all clinical studies are shown in Table S8 of the ESM. Further plots, showing the predictions of all available idarucizumabdabigatran studies that include coagulation measurements, are presented in Figs. S11-S14 of the ESM.

\subsection{Prediction of Dabigatran Reversal Scenarios}

The final models were used to predict different dabigatran reversal scenarios. The results of those predictions are shown in Fig. 7. The recommended dosing regimen of idarucizumab was compared to a standard hemodialysis protocol, regarding their effects on dabigatran plasma concentrations and diluted thrombin time (Fig. 7a, b). Furthermore, administrations of idarucizumab as a bolus or a 2-h infusion were compared (Fig. 7c), to evaluate if longer infusion times could reduce the dabigatran rebound. In addition, combined treatments of idarucizumab and hemodialysis were predicted (Fig. 7d), to assess if hemodialysis could support idarucizumab treatment and reduce dabigatran rebound, and if 2500
Fig. 6 Coagulation times as functions of unbound sum dabigatran (unb sum DAB) plasma concentrations (left column), in representative effect-time plots (middle) during dabigatran etexilate (DE) treatment (220 mg twice a day (bid), for 3.5 days) followed by administration of $1000 \mathrm{mg}$ of idarucizumab (IDA) for dabigatran reversal (at $242 \mathrm{~h}$, arrow) in healthy Japanese individuals $[19,21]$ and physiologically based pharmacokinetic/pharmacodynamic (PBPK/PD) model performance in goodness-of-fit plots (right column). Clinically observed data are shown as dots; colored solid lines indicate the model predicted coagulation times. The line of identity is shown as a black solid line; the 0.8 - to 1.25 -fold bioequivalence limits are shown as black dotted lines; the 0.5- to 2.0-fold prediction acceptance limits are shown as dashed lines. $a P T T$ activated partial thromboplastin time, $d T T$ diluted thrombin time, ECT ecarin clotting time, $J P N$ Japanese, $o b s$ observed, $P D$ pharmacodynamics, pred predicted, $T T$ thrombin time

mg of idarucizumab combined with hemodialysis could be as effective as the standard idarucizumab dosing regimen of $2 \times 2500 \mathrm{mg}$.

The predictions show that administration of the standard dosing regimen of $2 \times 2500 \mathrm{mg}$ results in complete dabigatran reversal, while $4 \mathrm{~h}$ of hemodialysis reduce the dabigatran plasma concentration by approximately $60 \%$. Furthermore, the administration of $1 \times 2500 \mathrm{mg}$ idarucizumab as a 2-h infusion markedly reduces the rebound effect compared with the administration of $1 \times 2500 \mathrm{mg}$ as a bolus injection.
Fig. 5 Plasma concentrations of sum dabigatran (DAB) before, during, and after hemodialysis. Start of hemodialysis is indicated by arrows. a Dabigatran etexilate (DE) is administered three times in doses of 150 $\mathrm{mg}, 110 \mathrm{mg}$, and $75 \mathrm{mg}$. Blood flow rate $(\mathrm{BFR})=200 \mathrm{~mL} / \mathrm{min}$ during two 4-h hemodialysis sessions. b DE is administered four times in doses of $150 \mathrm{mg}$ bid. $\mathrm{BFR}=320 \mathrm{~mL} / \mathrm{min}$ during a 6-h hemodialysis. $\mathbf{c} \mathrm{DE}$ is administered six times in doses of $150 \mathrm{mg}$ bid. $\mathrm{BFR}=375 \mathrm{~mL} /$ min during a 3-h hemodialysis. d DE is administered three times in doses of $150 \mathrm{mg}, 110$ $\mathrm{mg}$, and $75 \mathrm{mg}$. BFR $=400 \mathrm{~mL} /$ min during two 4-h hemodialysis sessions. Clinically observed data are shown as dots; solid lines indicate the model prediction
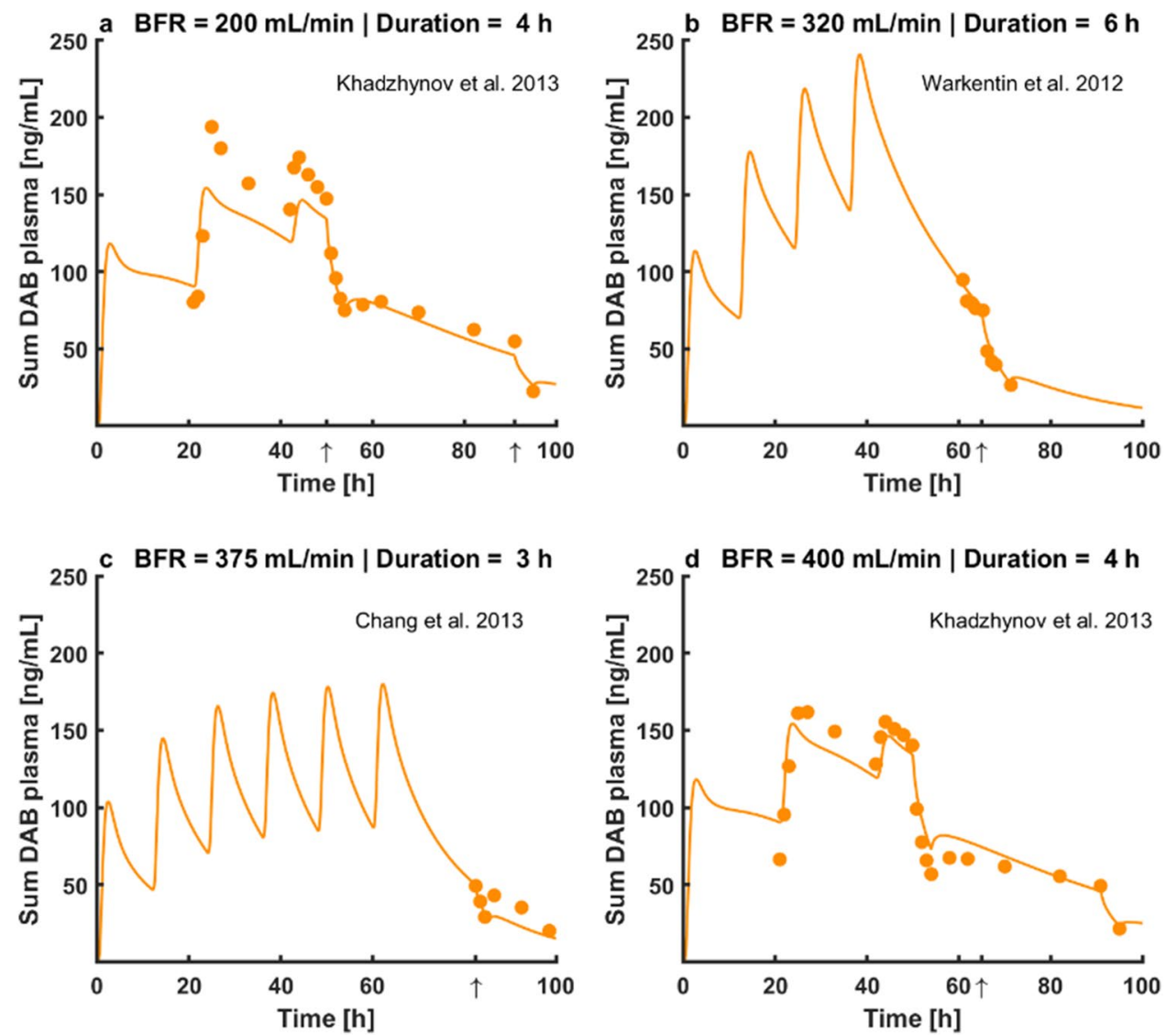

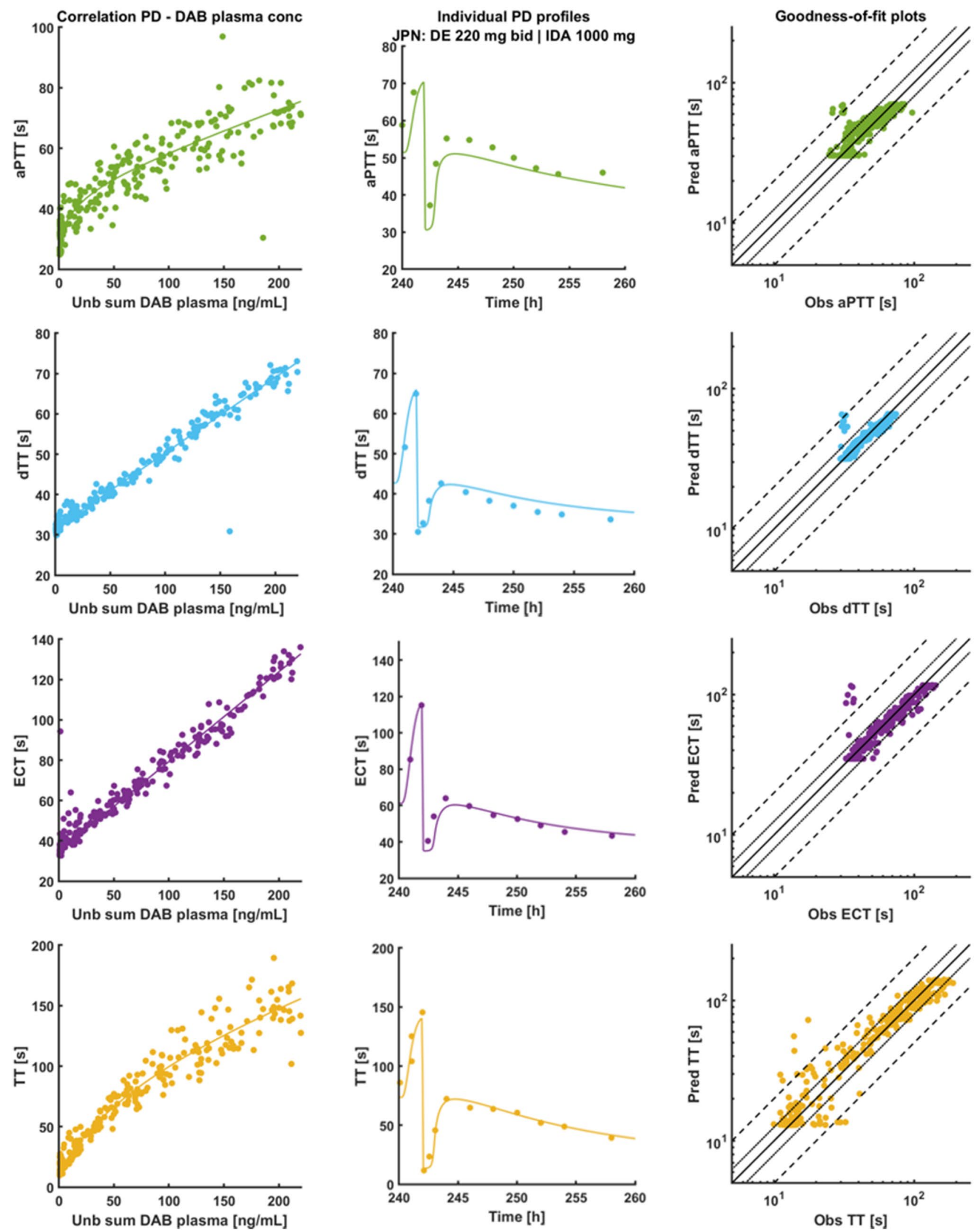
Fig. 7 Predicted dabigatran plasma concentrations (left column) and diluted thrombin times (dTT) (right column) for different reversal scenarios. Dabigatran etexilate (DE) is administered in doses of $220 \mathrm{mg}$ twice a day (bid). a Standard idarucizumab (IDA) dosing regimen of two bolus injections of $2500 \mathrm{mg}$. b 4-h hemodialysis with a blood flow rate (BFR) of $400 \mathrm{~mL} / \mathrm{min}$ and a dialysate flow rate (DFR) of $700 \mathrm{~mL} /$ min. c Comparison of different IDA administration protocols: application of $2500 \mathrm{mg}$ as a bolus injection (solid lines) vs application as a 2-h infusion (dashed lines). d 2-h infusions of $2500 \mathrm{mg}$ (dashed lines) or $3500 \mathrm{mg}$ (dotted lines) of IDA, applied in combination with a 6-h hemodialysis. The grey dotted line shows the threshold for complete dabigatran reversal of $20 \mathrm{ng} / \mathrm{mL}$. unb sum DAB unbound sum dabigatran
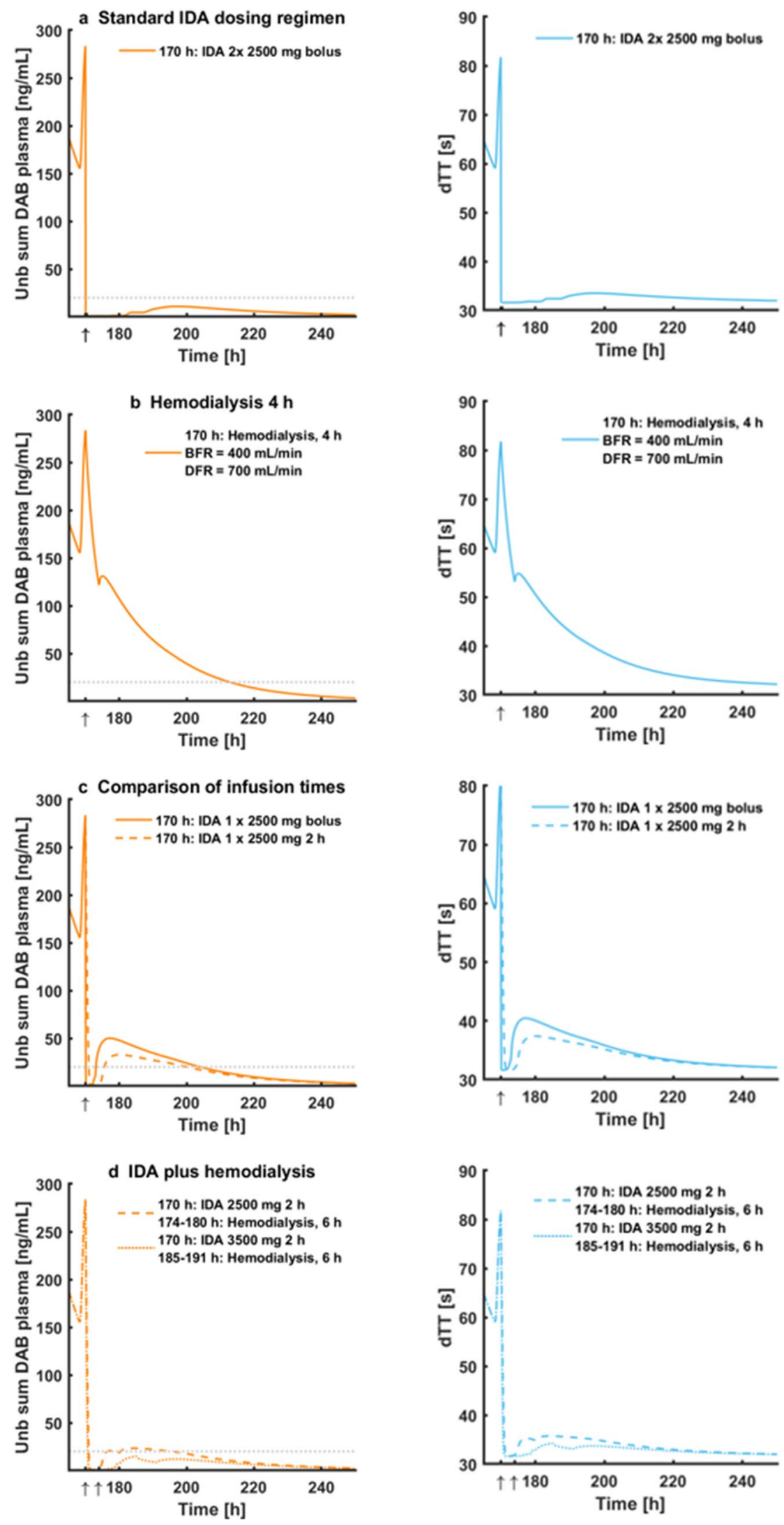
When hemodialysis is started after the 2-h infusion of $1 \times$ $2500 \mathrm{mg}$ (Fig. 7d), the rebound is further reduced and close to the threshold for complete reversal $(20 \mathrm{ng} / \mathrm{mL})$ [14]. To achieve complete reversal with a combined treatment of a single dose of idarucizumab and hemodialysis, the idarucizumab dose has to be elevated to $3500 \mathrm{mg}$.

\section{Discussion}

The presented idarucizumab PBPK model accurately describes and predicts plasma concentration-time profiles and fractions excreted to urine over the full range of doses and administration protocols reported in the literature. The model has been qualified to describe the PK and PD interactions of the large molecule idarucizumab with the small molecule dabigatran within the same PBPK modeling environment, and has been applied to explore dabigatran reversal with idarucizumab or hemodialysis in a PBPK/PD approach.

For a mechanistic description of the renal handling of idarucizumab, the kidney structure was extended by a tubule compartment with megalin-mediated transport from the tubule lumen into the renal intracellular endosome. This is to our knowledge the first PBPK modeling approach that includes megalin-mediated endocytosis into proximal tubular cells as a process involved in the renal handling of therapeutic proteins. In animals, it was demonstrated that renal dysfunction can lead to reduced expression of megalin via the release of inflammatory mediators [34-36]. In the presented analysis, a correlation between $\mathrm{CrCl}$ and the idarucizumab reabsorption via megalin was observed, decreasing the megalin activity with decreasing renal function. This correlation was implemented, to correctly describe the urinary excretion of idarucizumab in patients with different stages of renal impairment. Using the age-dependent physiological parameters in the PK-Sim ${ }^{\circledR}$ database and the reported actual $\mathrm{CrCl}$ to generate the individuals for the different studies, no further adjustment of the model was necessary to describe the pharmacokinetics of idarucizumab in renally impaired patients. Physiological characteristics of Japanese individuals, also provided in the modeling platform, were used to describe the pharmacokinetics of idarucizumab in Japanese populations. Yet, the idarucizumab plasma concentrations in Japanese volunteers were under-predicted throughout all simulated profiles, which could be significantly improved by reducing the parameter "GFR fraction" from 0.32 to 0.26 (both values are optimized) for the Japanese population. No explanation for these observed ethnic differences could be found in the literature.

Before establishing the interaction of idarucizumab and dabigatran, a previously published dabigatran PBPK model [27] was evaluated by predicting dabigatran plasma concentrations without co-administration of idarucizumab. The excellent model performance is summarized in goodness-offit plots presented in Fig. S10 of the ESM and MRD values are shown in Table S7 of the ESM.

The parameters to model the idarucizumab-dabigatran interaction were taken from the literature without any further adjustment. With this parameterization, the model successfully describes the drastic and complete reduction of dabigatran concentrations in the plasma of healthy subjects and patients, observed after idarucizumab application.

As it has been postulated that the pharmacokinetics of the idarucizumab complexes do not differ from those of idarucizumab alone [10], endosomal uptake and degradation of these complexes in the kidney were included into the model. During the breakdown of the complexes, dabigatran is released and the free dabigatran escapes endosomal degradation and is excreted with the urine [10]. So far, there is no information on the lysosomal handling of the small molecule dabigatran and how it is released back into the tubule. Therefore, a simple exocytotic transport of dabigatran from the endosome to the tubule was implemented to describe the observed dabigatran fraction excreted to urine. Dabigatran was not transported back into the systemic circulation in our model as this would result in a second plasma rebound, not matching the observed data.

The final idarucizumab-dabigatran PBPK/PD model adequately predicts the impact of dabigatran on aPTT, dTT, ECT, and TT as well as the effect of dabigatran reversal by idarucizumab on these coagulation markers. After successful evaluation, this model was used to predict different dabigatran reversal scenarios for a typical patient. Based on the population of the idarucizumab phase III study "RE-VERSE AD", the average patient in need of idarucizumab shows a $\mathrm{CrCl}$ of $62 \mathrm{~mL} / \mathrm{min}$. As the GFR has a significant impact on the pharmacokinetics of idarucizumab and dabigatran, it is important to consider the renal function during the modeling of idarucizumab and idarucizumab-dabigatran studies. Our predictions show that the standard idarucizumab dosing regimen of two 2500-mg bolus injections results in a complete and immediate reduction in dabigatran plasma concentrations, whereas a 4-h hemodialysis reduces dabigatran plasma concentrations by approximately $60 \%$. These findings are in accordance with current treatment recommendations, confirming the recommended idarucizumab regimen of 2 $\times 2500 \mathrm{mg}$. Compared to hemodialysis, administration of idarucizumab ensures immediate and complete dabigatran reversal and is therefore a safer and more convenient, but also more expensive therapy. Our results further suggest that longer idarucizumab infusion times have the potential to reduce dabigatran rebound and that a combination with hemodialysis could further suppress the rebound. This might be helpful for patients with moderate or severe renal impairment, where a stronger dabigatran rebound is expected, or in situations when there is not enough idarucizumab 
available and lower doses have to be administered. Additional clinical data are needed to corroborate this hypothesis. regulation or exceeds the permitted use, you will need to obtain permission directly from the copyright holder.To view a copy of this licence, visit http://creativecommons.org/licenses/by-nc/4.0/.

\section{Conclusions}

A comprehensive and mechanistic PBPK/PD model to study dabigatran reversal has been established, which includes whole-body PBPK modeling of idarucizumab, the idarucizumab-dabigatran interaction, dabigatran hemodialysis, the dabigatran plasma concentration-effect relationship, and the impact of renal impairment in these different scenarios. The presented idarucizumab PBPK model is the first published in the literature and the idarucizumab-dabigatran interaction model successfully predicts the changes in dabigatran plasma concentrations and the PD effects on coagulation times. Model building and evaluation of this PBPK/PD model are transparently documented, including the model performance for all clinical studies obtained from the literature. The presented model will be freely available in the Open Systems Pharmacology repository (https://www.opensystems-pharmacology.org) and can be applied to investigate and understand the pharmacokinetics and pharmacodynamics of idarucizumab and dabigatran as well as the outcomes of different dosing regimens. Furthermore, the thorough analysis and implementation of the impact of renal function on all aspects of this PBPK/PD model make it applicable as a tool to guide individual idarucizumab dosing in clinical practice, taking us one step closer to our ultimate goal of precision medicine.

Acknowledgement Open Access funding provided by Projekt DEAL.

\section{Compliance with Ethical Standards}

Funding The project has received support from the project "Opensource modeling framework for automated quality control and management of complex life science systems models" (OSMOSES), which is funded by the German Federal Ministry of Education and Research (BMBF, Grant ID 031L0161C).

Conflict of interest Bernd Meibohm has provided consulting services to Boehringer Ingelheim, the maker of dabigatran and idarucizumab, on unrelated development projects. Laura Maria Fuhr, Nina Hanke, and Thorsten Lehr have no conflicts of interest that are directly relevant to the content of this article.

Open Access This article is licensed under a Creative Commons Attribution-NonCommercial 4.0 International License, which permits any non-commercial use, sharing, adaptation, distribution and reproduction in any medium or format, as long as you give appropriate credit to the original author(s) and the source, provide a link to the Creative Commons licence, and indicate if changes were made. The images or other third party material in this article are included in the article's Creative Commons licence, unless indicated otherwise in a credit line to the material. If material is not included in the article's Creative Commons licence and your intended use is not permitted by statutory

\section{References}

1. Boehringer Ingelheim Pharmaceuticals Inc. Full prescribing information: Pradaxa. 2015 https://www.accessdata.fda.gov/drugsatfda _docs/label/2015/022512s0281bl.pdf. Accessed 4 Nov 2019.

2. Dansirikul C, Lehr T, Liesenfeld KH, Haertter S, Staab A. A combined pharmacometric analysis of dabigatran etexilate in healthy volunteers and patients with atrial fibrillation or undergoing orthopaedic surgery. Thromb Haemost. 2012;107:775-85.

3. Stangier J, Rathgen K, Stähle H, Gansser D, Roth W. The pharmacokinetics, pharmacodynamics and tolerability of dabigatran etexilate, a new oral direct thrombin inhibitor, in healthy male subjects. Br J Clin Pharmacol. 2007;64:292-303.

4. Stangier J, Clemens A. Pharmacology, pharmacokinetics, and pharmacodynamics of dabigatran etexilate, an oral direct thrombin inhibitor. Clin Appl Thromb Hemost. 2009;15(Suppl. 1):9S-16S.

5. Wilson J-AS, Goralski KB, Soroka SD, Morrison M, Mossop P, Sleno L, et al. An evaluation of oral dabigatran etexilate pharmacokinetics and pharmacodynamics in hemodialysis. J Clin Pharmacol. 2014;54:901-9.

6. Stangier J, Feuring M. Using the HEMOCLOT direct thrombin inhibitor assay to determine plasma concentrations of dabigatran. Blood Coagul Fibrinolysis. 2012;23:138-43.

7. Douxfils J, Dogné J-M, Mullier F, Chatelain B, Rönquist-Nii Y, Malmström RE, et al. Comparison of calibrated dilute thrombin time and aPTT tests with LC-MS/MS for the therapeutic monitoring of patients treated with dabigatran etexilate. Thromb Haemost. 2013;110:543-9.

8. Blech S, Ebner T, Ludwig-Schwellinger E, Stangier J, Roth W. The metabolism and disposition of the oral direct thrombin inhibitor, dabigatran, in humans. Drug Metab Dispos. 2008;36:386-99.

9. Khadzhynov D, Wagner F, Formella S, Wiegert E, Moschetti V, Slowinski T, et al. Effective elimination of dabigatran by haemodialysis: a phase I single-centre study in patients with end-stage renal disease. Thromb Haemost. 2013;109:596-605.

10. Eikelboom JW, Quinlan DJ, van Ryn J, Weitz JI. Idarucizumab: the antidote for reversal of dabigatran. Circulation. 2015;132:2412-22.

11. Ryman JT, Meibohm B. Pharmacokinetics of monoclonal antibodies. CPT Pharmacometrics Syst Pharmacol. 2017;6:576-88.

12. Meibohm B, Zhou H. Characterizing the impact of renal impairment on the clinical pharmacology of biologics. J Clin Pharmacol. 2012;52(1 Suppl.):54S-62S.

13. Christensen EI, Birn H. Megalin and cubilin: synergistic endocytic receptors in renal proximal tubule. Am J Physiol Ren Physiol. 2001;280:562-73.

14. Glund S, Stangier J, van Ryn J, Schmohl M, Moschetti V, Haazen $\mathrm{W}$, et al. Effect of age and renal function on idarucizumab pharmacokinetics and idarucizumab-mediated reversal of dabigatran anticoagulant activity in a randomized, double-blind, crossover phase Ib study. Clin Pharmacokinet. 2017;56:41-54.

15. Reilly PA, Lehr T, Haertter S, Connolly SJ, Yusuf S, Eikelboom JW, et al. The effect of dabigatran plasma concentrations and patient characteristics on the frequency of ischemic stroke and major bleeding in atrial fibrillation patients: the RE-LY Trial (Randomized Evaluation of Long-Term Anticoagulation Therapy). J Am Coll Cardiol. 2014;63:321-8.

16. Paré G, Eriksson N, Lehr T, Connolly S, Eikelboom J, Ezekowitz $\mathrm{MD}$, et al. Genetic determinants of dabigatran plasma levels and their relation to bleeding. Circulation. 2013;127:1404-12. 
17. Boehringer Ingelheim Pharmaceuticals Inc. BI Trial No. 1321.2 Randomised, double-blind, placebo-controlled, two-way crossover phase Ib study to investigate the safety, tolerability, pharmacokinetics and pharmacodynamics of BI 655075 and to establish the efficacy of BI 655075 in reversal of dabigatran anticoagulant activity in volunteers. EudraCT: 2013-003616-52. 2014.

18. Boehringer Ingelheim Pharmaceuticals Inc. BI Trial No. 1321.1 Randomised, double-blind, placebo-controlled phase I study in healthy male volunteers to investigate safety, tolerability and pharmacokinetics of single rising doses of BI 655075 (part 1) and to explore the dose of BI 655075 effective to reverse dabigatran anticoagulant activity (part 2). EudraCT: 2012-003611-66. 2014.

19. Boehringer Ingelheim Pharmaceuticals Inc. BI Trial No. 1321.5 Randomised, double-blind within dose groups, placebocontrolled phase I trial in healthy Japanese male volunteers to investigate safety, tolerability and pharmacokinetics of different doses of BI 655075 (part 1) and to explore the effective dose of BI 655075 to reverse dabigatran anticoagulant activity (part 2). NCT Number: NCT02028780. 2015.

20. Glund S, Moschetti V, Norris S, Stangier J, Schmohl M, van Ryn $\mathrm{J}$, et al. A randomised study in healthy volunteers to investigate the safety, tolerability and pharmacokinetics of idarucizumab, a specific antidote to dabigatran. Thromb Haemost. 2015;113:943-51.

21. Yasaka M, Ikushima I, Harada A, Imazu S, Taniguchi A, Norris S, et al. Safety, pharmacokinetics and pharmacodynamics of idarucizumab, a specific dabigatran reversal agent in healthy Japanese volunteers: a randomized study. Res Pract Thromb Haemost. 2017;1:202-15.

22. Glund S, Coble K, Gansser D, Stangier J, Hoermann K, Pollack $\mathrm{CV}$, et al. Pharmacokinetics of idarucizumab and its target dabigatran in patients requiring urgent reversal of the anticoagulant effect of dabigatran. J Thromb Haemost. 2019;17:1319-28.

23. Glund S, Stangier J, Schmohl M, Gansser D, Norris S, van Ryn $\mathrm{J}$, et al. Safety, tolerability, and efficacy of idarucizumab for the reversal of the anticoagulant effect of dabigatran in healthy male volunteers: a randomised, placebo-controlled, double-blind phase 1 trial. Lancet. 2015;386:680-90.

24. Warkentin TE, Margetts P, Connolly SJ, Lamy A, Ricci C, Eikelboom JW. To the editor: recombinant factor VIIa (rFVIIa) and hemodialysis to manage massive dabigatran-associated. Blood. 2012;119:2172-4.

25. Chang DN, Dager WE, Chin AI. Removal of dabigatran by hemodialysis. Am J Kidney Dis. 2013;61:487-9.
26. Balazki P, Schaller S, Eissing T, Lehr T. A quantitative systems pharmacology kidney model of diabetes associated renal hyperfiltration and the effects of SGLT inhibitors. CPT Pharmacometrics Syst Pharmacol. 2018;7:788-97.

27. Moj D, Maas H, Schaeftlein A, Hanke N, Gómez-Mantilla JD, Lehr T. A comprehensive whole-body physiologically based pharmacokinetic model of dabigatran etexilate, dabigatran and dabigatran glucuronide in healthy adults and renally impaired patients. Clin Pharmacokinet. 2019;58:1577-93.

28. Sié P. Spotlight on idarucizumab and its potential for the reversal of anticoagulant effects of dabigatran. Drug Des Devel Ther. 2016;10:1683-9.

29. Liesenfeld K-H, Staab A, Härtter S, Formella S, Clemens A, Lehr T. Pharmacometric characterization of dabigatran hemodialysis. Clin Pharmacokinet. 2013;52:453-62.

30. Liesenfeld K-H, Schäfer HG, Trocóniz IF, Tillmann C, Eriksson BI, Stangier J. Effects of the direct thrombin inhibitor dabigatran on ex vivo coagulation time in orthopaedic surgery patients: a population model analysis. Br J Clin Pharmacol. 2006;62:527-37.

31. Pollack CV, Reilly PA, Eikelboom J, Glund S, Verhamme P, Bernstein RA, et al. Idarucizumab for dabigatran reversal. N Engl J Med. 2015;373:511-20.

32. Härtter S, Sennewald R, Nehmiz G, Reilly P. Oral bioavailability of dabigatran etexilate $\left(\operatorname{Pradaxa}{ }^{\circledR}\right)$ after co-medication with verapamil in healthy subjects. Br J Clin Pharmacol. 2013;75:1053-62.

33. Liesenfeld K-H, Lehr T, Dansirikul C, Reilly PA, Connolly SJ, Ezekowitz MD, et al. Population pharmacokinetic analysis of the oral thrombin inhibitor dabigatran etexilate in patients with nonvalvular atrial fibrillation from the RE-LY trial. J Thromb Haemost. 2011;9:2168-75.

34. Gorriz JL, Martinez-Castelao A. Proteinuria: detection and role in native renal disease progression. Transplant Rev (Orlando). 2012;26:3-13

35. Marzolo M, Farfán P. New insights into the roles of megalin/ LRP2 and the regulation of its functional expression. Biol Res. 2011;44:89-105.

36. Odera K, Goto S, Takahashi R. Age-related change of endocytic receptors megalin and cubilin in the kidney in rats. Biogerontology. 2007;8:505-15.

37. Schiele F, van Ryn J, Canada K, Newsome C, Sepulveda E, Park J, et al. A specific antidote for dabigatran: functional and structural characterization. Blood. 2013;121:3554-62. 